\title{
Analytical Multilayer Model Revisited for High Atomic Numbers at Low Voltage.
}

\author{
Claude Merlet
}

GM, CNRS, Université de Montpellier II, Pl. E. Bataillon, 34095 Montpellier cedex 5, France

Electron probe micro-analysis (EPMA), originally developed to determine the composition of the bulk samples at the micron scale, has become a well established technique to determine the compositions and the thickness of thin multilayer deposited on a substrate [1-4]. This technique can be used to determine the film thickness in a range of a few micrometers to a monolayer. By varying the accelerating voltage, and thus the excitation depths, the different layers can be analyzed. Typical accelerating voltages of 5$40 \mathrm{kV}$ have excitation depths from $0.2-10 \mu \mathrm{m}$. The thin-multilayer method by EPMA is based on the comparison of the ratios of x-ray intensities (k-ratio) emitted by the elements of the various layers to those emitted from bulk standards under same experimental conditions. To convert the measured k-ratio from elements of the layers in thicknesses and compositions, a multilayer model requires an accurate description of the x-ray depth distribution $(\phi(\rho z))$ from which the emitted x-ray intensities are calculated. It is worth noting that for an element corresponds a specific $\phi(\rho z)$ distribution which varies with accelerating voltages and with the thicknesses and compositions of the layers.

Analytical $\phi(\rho z)$ models, which have been successfully used in many applications, are known to be less accurate when there are large differences of atomic number between the different layers. In analytical approaches, in a stratified sample and for each element of layers, the $\phi(\rho z)$ distributions are built by using a weighting procedure according to the layers combinations. The simplest case corresponds to a thin layer/substrate with pure elements whose atomic numbers are neighboring. For this simple case the electron scattering and the $\mathrm{x}$-ray generation are similar as in bulk sample and the $\phi(\rho z)$ distributions from thin layer/substrate are similar to bulk sample. When the deviation of the atomic number between thin layer and substrate increases, the difference in electron scattering properties between thin layer and substrate increases and consequently a change in the $\phi(\rho z)$ distributions comparatively to bulk samples. Similarly the thickness of the thin layer relatively to the $\phi(\rho z)$ distributions can vary between two extremes: extremely thin or extremely thick. In the first case, the $\phi(\rho z)$ distributions from layer/substrate correspond to a bulk with the composition of the substrate, and in the second case, to a bulk with the composition of the layer. Analytical $\phi(\rho z)$ distributions are based on mathematical functions that are set by a number of parameters that depend on the experimental conditions and of the sample. One of them, the surface ionization $\phi(0)$ appears explicitly in most analytical $\phi(\rho z)$ distribution. For surface layers, calculations of x-ray intensities largely depend on the adopted $\phi(0)$ values, especially for thinner films, or when the $\mathrm{x}$-ray range is large relatively the film thickness.

Improvement in the thin-film analytical code XFILM [3-4] at low accelerating voltage and low overvoltage for high atomic numbers was done by revisited analytical parameterizations of the $\phi(\rho z)$ distribution. The reliability of thin-film programs has usually been assessed by comparing measured and calculated $k$-ratios from well-characterized thin-film samples. Depending of the sample structure, we can expect with thin-multilayer method an accuracy in the thickness determination better than $5 \%$ even when there are large differences in atomic number between the different layers. However, deviations in the thickness determination between experimental measurements and the thin-film analytical code XFILM have been observed at low accelerating voltage for $M$ lines of high atomic numbers. As 
example, Murata and Sugiyama [5] have measured Au films with a thickness range of 0.2-100nm on Si substrates at different accelerating voltage. For these measurements, the agreement between calculated and experimental k-ratios was good except at $3.5 \mathrm{kV}$ for the thicknesses range from 1-10 nm where XFILM overestimated the k-ratios. For $\mathrm{M}$ lines of high atomic numbers elements, low accelerating voltage require to work at low overvoltage and consequently in a steep region of the x-ray production cross section and of the surface ionization. To improve the analytical model at low voltage for $\mathrm{M}$ lines, recent measurements done with high atomic numbers films deposited onto various substrates and also onto self-supporting thin $\mathrm{C}$ backing films were used [6,7]. Measurements performed simultaneously on self-supporting and substrates allow to reach the x-ray production cross section [6] as well as the surface ionization [7]. In addition, the measurements on the various substrates allow to have information about scattering properties and also to check the weighting procedure for layer/substrate used in the analysis code. These data were used among others to improve the analytical formulation of the surface ionization [8] at low accelerating voltage for $M$ lines. The surface ionization has a large impact in the k-ratio calculation at low accelerating voltage for nanometers thicknesses. In this range of thicknesses at low accelerating voltage, high atomic numbers layer increases significantly the $\phi(0)$ value and then the intensity. All uncertainties in the $\phi(0)$ calculation impact directly the k-ratio result.

With the new optimized analytical parameterizations at low voltage, the improvement of the intensity calculation for high atomic numbers films is significant. As example, for an Au/Si layer of $1.5 \mathrm{~nm}$ thickness, with the new parameterizations, deviations between calculated and experimental intensities are reduced to less than $5 \%$ for voltage in the range $4.5-3 \mathrm{kV}$. It is worth noting that accurate measurements of intensities at low voltage and low overvoltage are not straightforward because surface contamination or slight uncertainties in the accelerating voltage can give large deviations [9]. For accelerating voltage higher than $4.5 \mathrm{kV}$, the uncertainties are in a range of $2 \%$ and are comparable to previous calculation. It is important to remember that, as the thickness is obtained with the relative intensities (k-ratios), a deviation can come from the intensity calculation of the layer or from the bulk standard. For thin layers, and in contrast to bulk samples, uncertainties in atomic parameters are not compensated when using standards. For Bulk $\mathrm{Au}$, the modification of the analytical parameterizations improves slightly the intensity calculation at low voltage and the agreement with the experimental measurements are good over all the accelerating voltage; the maximum deviation appears at low voltage $(4 \%$ at $3 \mathrm{kV})$ and is mainly the result of the measurement.

\section{References:}

[1] GF Bastin and HJM Heijligers, X-ray spectrometry 29, 212 (2000). [2] J.L. Pouchou, and F. Pichoir, Rech Aerosp 5, 349 (1984).

[3] C. Merlet, Proc. of Microbeam Analysis, Edited by E. S. Etz, VCH Publishers, 203, (1995).

[4] X. Llovet and C. Merlet, Micros. Microanal. 16, 21(2010).

[5] K Murata and K Sugiyama, J Appl Phys 54, 1110 (1989).

[6] C. Merlet, X. Llovet and F. Salvat, Phys. Rev. A 78022704 (2008).

[7] C. Merlet and X. Llovet, X-Ray Spectrom. 40, 47 (2011).

[8] C. Merlet, X-Ray Spectrom. 21, 229 (1992).

[9] C. Merlet and X. Llovet, IOP Conf. Ser. Mater. Sci. Eng., 32, 012016 (2012) 